\title{
Modélisation de l'irradiation solaire au pas de temps de l'heure
}

\author{
G. Boch, E. Boileau et C. Bénard \\ C.N.R.S. Ecole Supérieure d'Electricité, Laboratoire des Signaux et Systèmes, Plateau du Moulon, 91190 Gif sur Yvette, France \\ (Reçu le 16 décembre 1980, révisé le 5 février 1981, accepté le 9 février 1981)
}

\begin{abstract}
Résumé. - Le processus constitué par l'irradiation globale reçue à Trappes et intégrée heure par heure a été étudié mois par mois. Après stationnarisation par construction d'une variable réduite, il a été constaté qu'une séparation en deux types de journées (d'après le signe de la valeur intégrée sur la journée de la variable réduite) conduisait à une modélisation satisfaisante. Ce modèle fournit des simulations de l'irradiation horaire globale sur une journée, ainsi que des prévisions à l'échelle de l'heure.
\end{abstract}

\begin{abstract}
The process of global irradiation received at Trappes and integrated hour by hour has been studied month by month. After stationnarisation by transformation to a reduced variable, separation in two kinds of daily series (according to the sign of the daily integrated value of the reduced variable) has led to a good model. This model yields daily simulations of the considered irradiations, and forecasts an hour ahead.
\end{abstract}

1. Introduction. - Cette étude concerne l'irradiation globale $\left({ }^{1}\right)$ au pas de temps de l'heure (c'est-àdire la valeur instantanée de l'irradiation globale intégrée sur 1 heure, et ceci heure par heure), de $4 \mathrm{~h}$ du matin à $8 \mathrm{~h}$ du soir (temps solaire vrai), soient 16 données journalières. Elle s'intercale entre des études à pas de temps plus long : la journée [1-5] et à pas de temps plus fin : la minute [6]. Une autre étude a déjà été faite au pas de temps de l'heure, mais à partir de variables différentes [7].

Nous avons travaillé, à partir de mesures fournies par le centre météorologique de Trappes, qui correspondent à la période du 1 er janvier 1971 au 31 décembre 1975 , soient 5 années de données.

On peut donc considérer chaque journée $j$ de ces 5 années $(j$ de 1 à 1826) comme une épreuve $(\omega)$ et l'irradiation globale au pas de temps de l'heure pour la journée considérée comme la suite aléatoire associée à l'épreuve $(\omega)$.

On définit donc la variable $I(m, j)$ avec :

$j$ : numéro du jour considéré ou indice de l'épreuve $(\omega)$

$m$ : variable temps représentant le numéro de l'heure, ici, de 1 à $16(m=1$ représentant l'heure de $4 \mathrm{~h}$ à $5 \mathrm{~h}, m=2$ de $5 \mathrm{~h}$ à $6 \mathrm{~h}$, etc...). $I(m, j)$ est donc la

$\left({ }^{1}\right)$ Irradiation globale : somme des irradiations solaires directe, et diffuse reçues sur une surface horizontale à partir d'un angle solide $2 \pi$. valeur du $m$-ième élément de la suite aléatoire associée au jour $j$.

Le but de l'étude est la modélisation de la variable $I(m, j)$ ou plutôt d'une variable déduite de celle-ci (pour satisfaire les contraintes qu'impose la modélisation).

On utilisera le modèle obtenu pour la simulation de journées au pas de temps de l'heure : ces journées simulées peuvent servir à l'optimisation de systèmes de stockage de l'énergie solaire ayant une inertie de l'ordre de l'heure. Le même modèle nous fournira ensuite des prévisions à l'heure (c'est-à-dire des prévisions de l'irradiation de l'heure prochaine à partir de l'irradiation mesurée pendant les heures précédentes), ce qui peut être intéressant pour les capteurs adaptatifs.

2. Choix de la variable et examen de sa stationnarité. - 2.1 NÉCESSITÉ D'UNE VARIABLE ALÉATOIRE STATIONNAIRE. - Pour effectuer la modélisation, on travaillera à partir des coefficients de corrélation, ce qui nécessite d'avoir une variable aléatoire stationnaire au second ordre $\left({ }^{2}\right)$ (sinon ces coefficients perdent toute signification car dépendant de l'origine des temps).

(2) Rappelons qu'une variable aléatoire est dite stationnaire au second ordre si ses moments d'ordre un et deux sont invariants par changement d'origine du temps ; la valeur moyenne ne dépend pas de l'époque pendant laquelle on la calcule et il en est de même pour ses coefficients de corrélation. 
Il est évident que $I(m, j)$ n'est pas stationnaire même au premier ordre, en effet, la figure 1 représente $\langle I(m, j)\rangle j$ en fonction de $m$.

$$
\langle I(m, j)\rangle_{j}=\frac{1}{1826} \sum_{j=1}^{1826} I(m, j)
$$

représente la moyenne de $I(m, j)$ pour une heure donnée $m$, prise sur tous les jours des 5 années considérées.

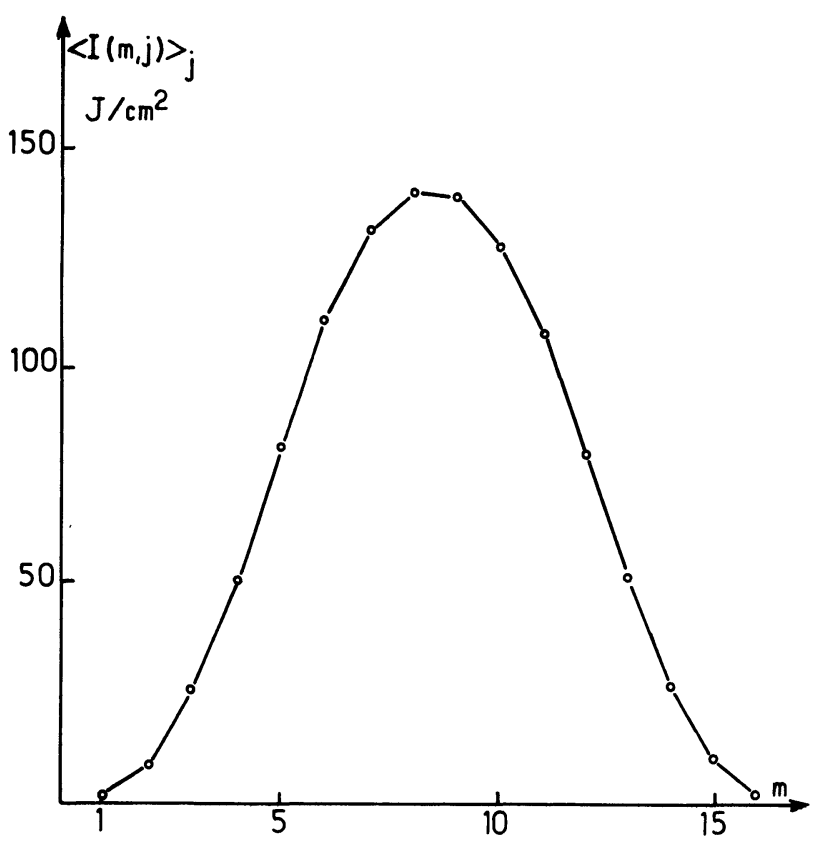

Fig. 1. - Valeur moyenne de $I(m, j)$, pour une heure donnée $m$, prise sur toutes les journées des cinq années.

[Mean value of $I(m, j)$, for a fixed hour $m$, calculated with all the days in the 5 years.]

On voit une très nette évolution de $\langle I(m, j)\rangle_{j}$ en fonction de $m$, ce qui prouve bien la non-stationnarité de $I(m, j)$. De même, la variance dépend évidemment de l'heure choisie, $m$.

Il est classique de supprimer les non-stationnarités de la moyenne et de la variance en se ramenant à un processus centré et de variance unité, mais les suites journalières que nous étudions ont des propriétés statistiques qui dépendent évidemment de la saison. On sait bien, par exemple, qu'en été, les journées sont en moyenne plus ensoleillées qu'en hiver, et ce serait une erreur de mélanger toutes les journées, c'est-à-dire de considérer les $j$ tous d'un seul bloc. On a effectué une séparation mois par mois. On peut admettre en effet qu'il n'y aura pas une nette évolution du comportement statistique au cours d'un mois donné.

2.2 SÉPARATION MOIS PAR MOIS. - On étudiera donc les 12 mois de l'année séparément, et pour simplifier les calculs, on a considéré que les mois auraient tous 30 jours (on a reporté le 31 janvier et le $1^{\text {er }}$ mars sur le mois de février, supprimé le 31 mai, 31 juillet,
31 août, 31 octobre et 31 décembre pour toutes les années et supprimé également le 29 février 1972).

Pour un mois donné $k$ (de 1 à 12), on a donc un ensemble $E_{k}$ de 150 jours de données $(5 \times 30)$, et on définit pour chaque heure $m$ (de 1 à 16), l'irradiation globale moyenne de l'heure $m$ pour le mois $k$ par :

$$
\bar{I}(m, k)=\frac{1}{150} \sum_{M=1}^{5} \sum_{j=j_{1}}^{j_{2}} I(m, j)=\left\langle I(m, j\rangle_{j \in E_{k}}\right.
$$

avec

$$
\left\{\begin{array}{l}
j_{1}=30[k+12(M-1)]-29 \\
j_{2}=30[k+12(M-1)]
\end{array}\right.
$$

De même, on définit l'écart-type pour un mois $k$ et une heure $m$ par :

$$
\sigma(m, k)=\left\{\frac{1}{150} \sum_{M=1}^{5} \sum_{j=j}^{j_{2}}[I(m, j)-\bar{I}(m, k)]^{2}\right\}^{1 / 2}
$$

qui représente pour 1 mois donné et une heure donnée la dispersion de l'ensoleillement des journées considérées autour de la moyenne. On a représenté sur les figures $2 a, b$ et $c$ les journées moyennes pour les mois de mars $(k=3)$, juin $(k=6)$ et octobre $(k=10)$, ainsi que, de part et d'autre de chacune de ces courbes, $\sigma(m, k)$. A partir de ces suites, $\bar{I}(m, k)$ et $\sigma(m, k)$, on définit, pour chaque jour $j$ d'un mois $k$, et pour chaque heure $m$, la variable réduite :

$$
Y(m, j)=\frac{I(m, j)-\bar{I}(m, k)}{\sigma(m, k)}
$$

changement de variable classique qui donne un processus centré et normé (variance unité).

En réalité, $Y(m, j)$ n'est pas vraiment centré, en effet, il y a une évolution continue de la moyenne de $Y(m, j)$ définie par (3) : par exemple, si on calcule cette moyenne sur les premiers jours de mars $(k=3)$, on trouve une moyenne nettement négative, si on calcule la même moyenne, mais sur les derniers jours de mars, on trouve une moyenne nettement positive. L'idéal serait de définir une journée moyenne non plus mois par mois, mais jour par jour; compte tenu des données que nous possédons, ceci est impossible, en effet, pour chaque journée, nous n'avons que 5 épreuves, ce qui est insuffisant pour calculer une moyenne. Nous restons donc dans le cadre de 1 journée moyenne par mois.

2.3 Examen DE la Stationnarité. - Nous avons examiné si le coefficient de corrélation d'ordre $p$ de $Y(m, j), p$ quelconque, était indépendant de $m$ (pour prouver que $Y(m, j)$ est bien stationnaire du $2^{\mathrm{e}}$ ordre). Nous estimons le coefficient de corrélation d'ordre $p$ pour l'heure $m$ et pour 1 mois donné $k$ par :

$$
r_{p, m}=\langle Y(m, j) Y(m+p, j)\rangle_{j \in E_{k}} .
$$




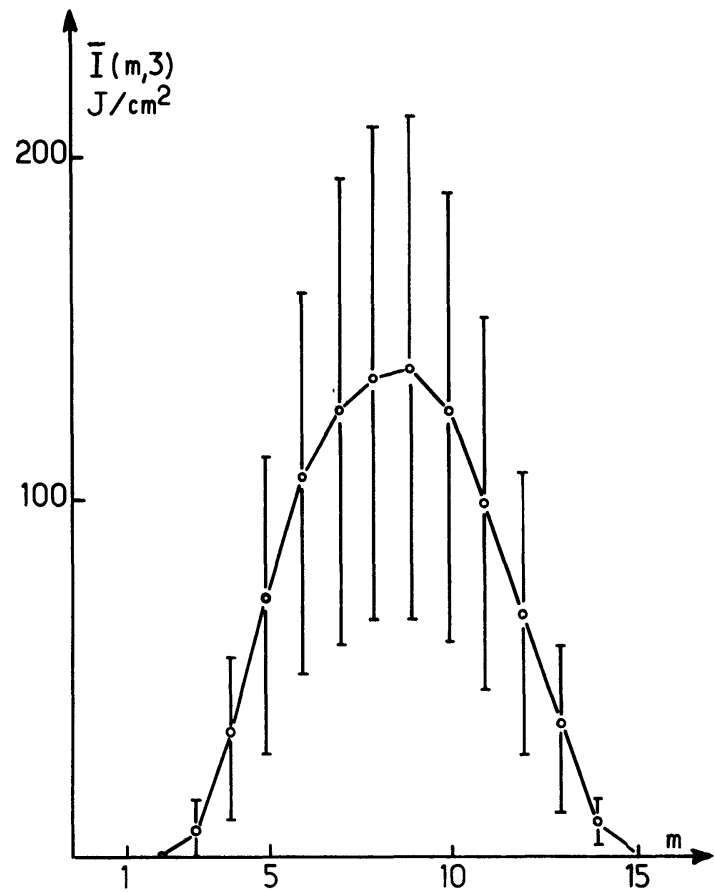

a)

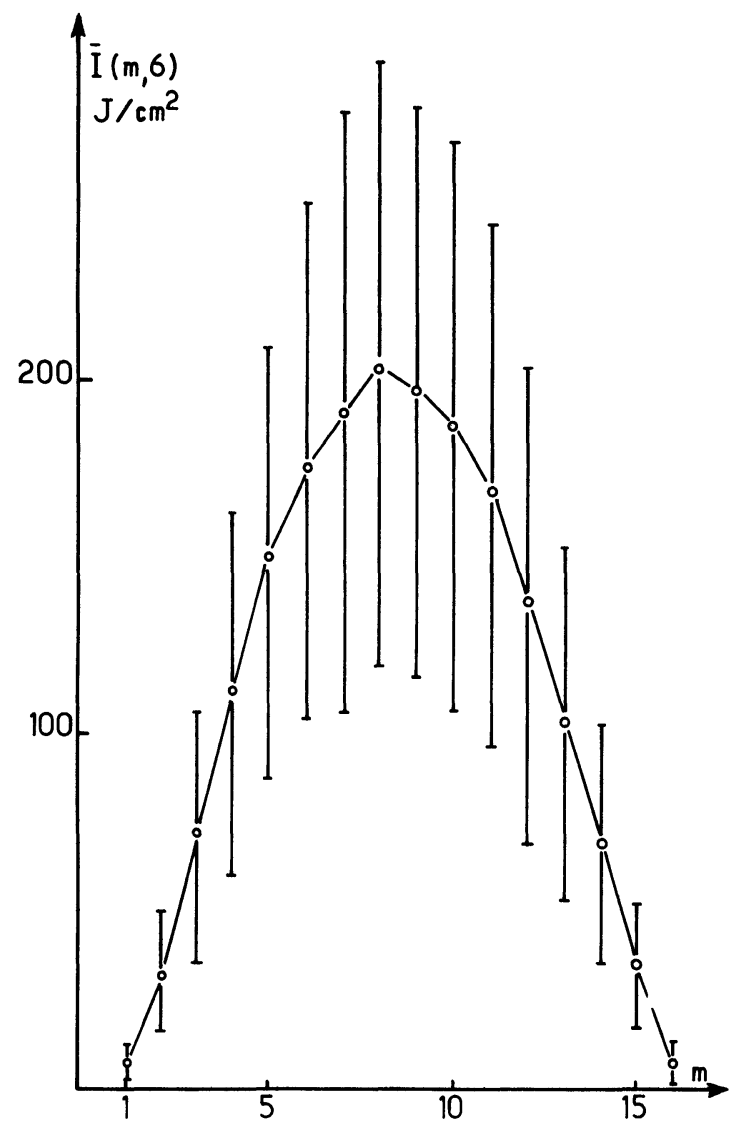

b)

Fig. $2 a, b, c$. - Valeur moyenne de $I(m, j)$, pour une heure donnée $m$, prise sur les 150 journées d'un mois donné, en fonction de $m$, avec écart-type, pour les mois de mars $(a)$, juin $(b)$, et octobre $(c)$.

[Mean value and standard deviation of $I(m, j)$ calculated with the days of March $(a)$, June $(b)$ and October $(c)$.]

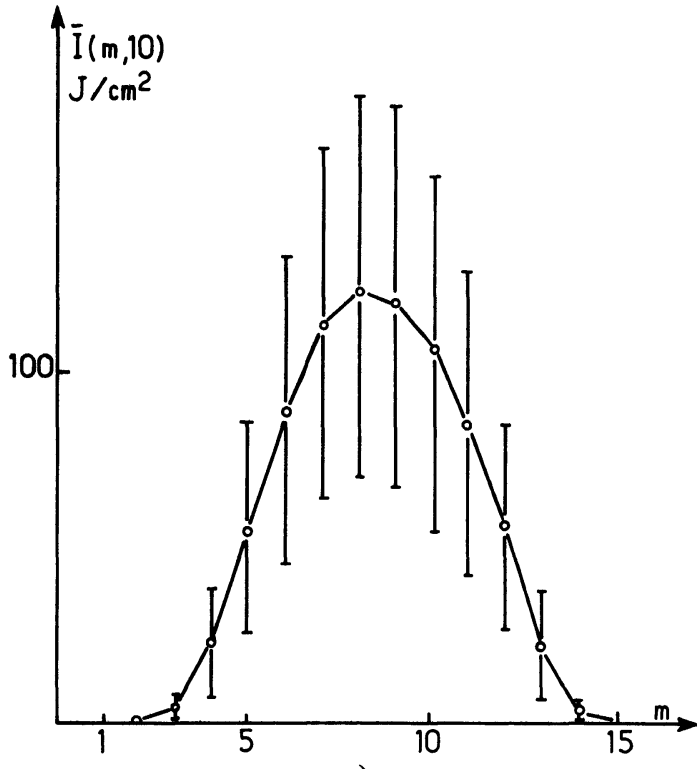

c)

L'écart-type relatif à cette estimation est de l'ordre de $1 / \sqrt{N}$ (cf. [8], $N$ étant le nombre de $j \in E_{k}: N=150$ ). Nous avons représenté sur la figure 3 , les coefficients de corrélation $r_{p, m}$ relatifs au mois d'avril $(k=4)$ pour $p=1$ et $p=2$.

Définissons $\rho_{p}$ par :

$$
\rho_{p}=\left\langle r_{p, m}\right\rangle_{m}
$$

(c'est-à-dire $: \rho_{p}=$ moyenne de $r_{p, m}$, moyenne prise sur $m$ ). Si le processus est stationnaire, $\rho_{p}$ est la meilleure approximation possible, avec nos données, du coefficient de corrélation d'ordre $p$.

On a alors représenté sur les figures précédentes. en trait plein, les droites $\rho_{p}+1 / \sqrt{N}$ et $\rho_{p}-1 / \sqrt{N}$. On remarque que presque tous les points $r_{p, m}$ sont situés entre les deux droites.

Comme $r_{p, m}$ est une approximation du coefficient de corrélation d'ordre $p$ à $1 / \sqrt{N}$ près, ceci prouve que l'hypothèse de stationnarité n'est pas contredite par le test.

3. Coefficients d'autocorrélation mois par mois. Pour un mois donné $k$, on a tracé $\rho_{p}$ pour $p=0, \ldots, 5$ (il n'est pas significatif d'aller plus loin du fait que l'on n'a que 16 heures de données par jour et que pour les mois d'hiver, les valeurs extrêmes sont nulles).

Pour tous les mois, on obtient des courbes qui ont l'allure d'exponentielles décroissantes, mais faiblement décroissantes (cf. Fig. 4). Une telle corrélation jusqu'à l'ordre 5 , (en moyenne : $\rho_{5}=0,6$ ) semble indiquer une très grande stabilité de $Y(m, j)$ au cours d'une journée. Essayons d'interpréter ce résultat autrement, imaginons une journée de très beau temps, ayant des $Y(m, j)$ positifs quelque soit $m$, l'apport de cette journée dans le calcul des $\rho_{p}$ est très élevé quelque soit l'ordre $p$. On arriverait à une conclusion identique pour une journée de très mauvais temps (avec des $Y(m, j)$ tous négatifs). En revanche, une 

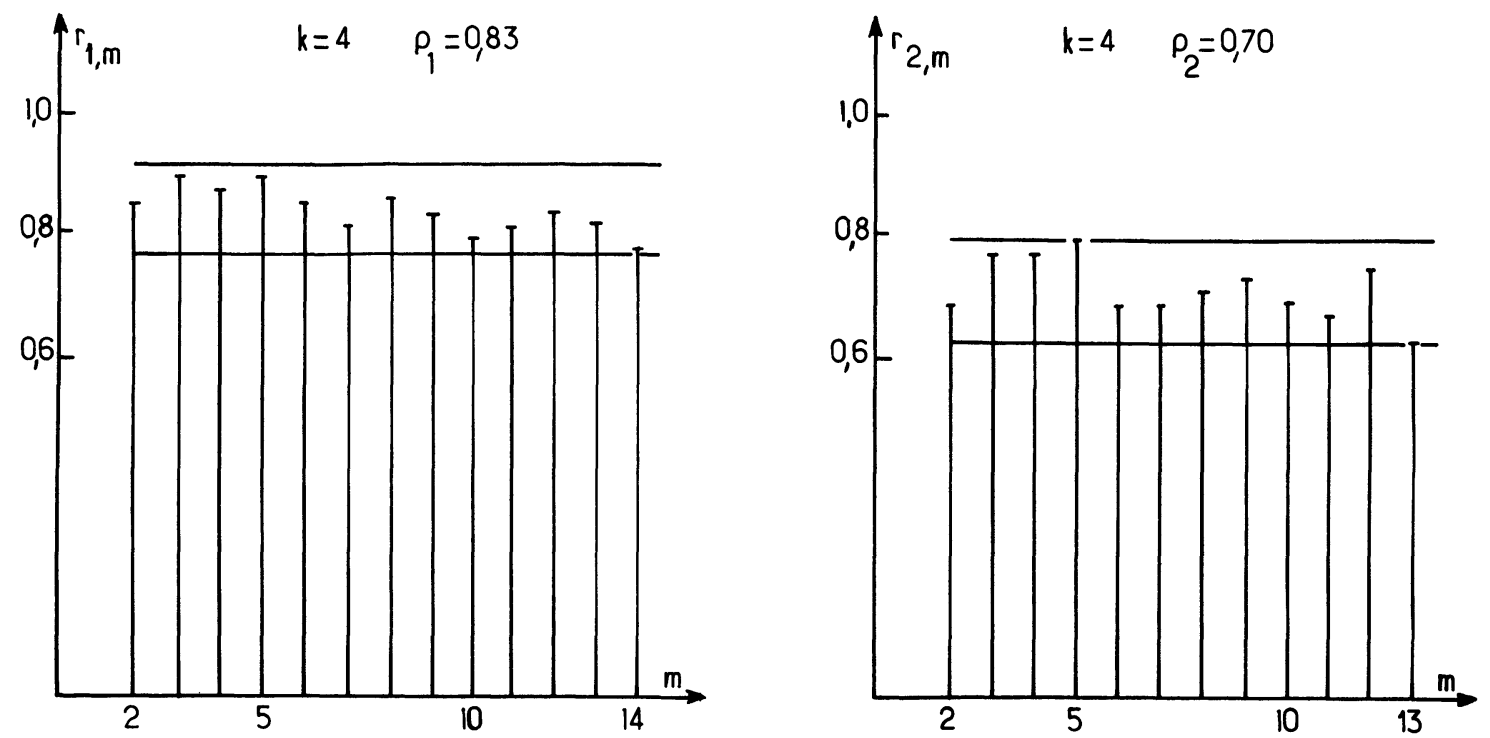

Fig. 3. - Examen de la stationnarité de la variable réduite $Y(m, j)$ relative au mois d'avril $(k=4)$ : évolution des coefficients de corrélation d'ordre 1 et 2 calculés à l'heure $m$ en fonction de $m$.

[Correlation coefficients (first and second order) of the reduced variable $Y(m, j)$ calculated at different hours $m$ in April $(k=4)$.]

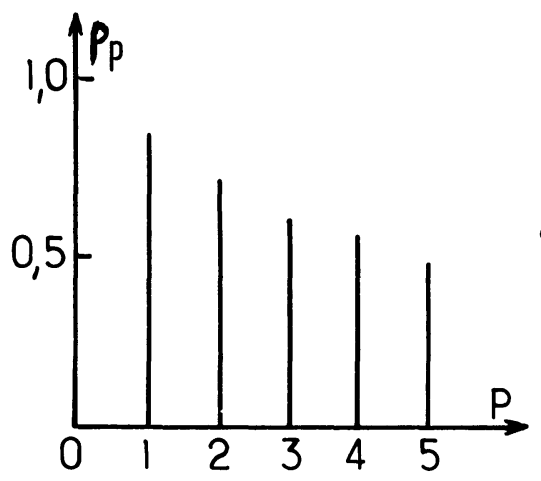

journée de temps moyen, c'est-à-dire avec des $Y(m, j)$ répartis autour de 0 , donnerait un apport corrélatif très faible par rapport aux deux cas précédents.

Ainsi, la décroissance lente de la fonction de corrélation obtenue peut résulter du mélange de sousensembles de types statistiques différents. Avant de

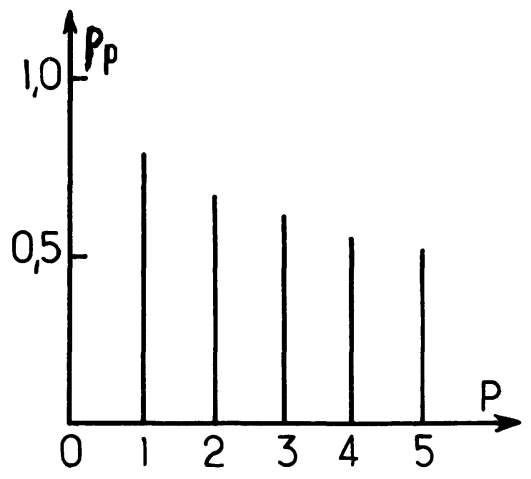
faire une quelconque séparation, nous allons d'abord vérifier si ce sont bien, comme nous venons de le supposer, les journées de temps extrême qui fournissent le plus grand apport corrélatif.

La précédente discussion nous a fait nous intéresser au comportement de $Y(m, j)$ au sein d'une journée $j$ donnée. Dans le prochain paragraphe, nous allons donc étudier pour une journée $j$ donnée, $\langle Y(m, j)\rangle_{m}$, (moyenne de $Y(m, j)$ prise sur les heures $m$ de la journée) ainsi que la répartition des $Y(m, j)$ autour de cette moyenne caractérisée par l'écart-type :

$$
\sigma_{Y}(j)=\left\{\frac{1}{16} \sum_{m=1}^{16}\left[Y(m, j)-\langle Y(m, j)\rangle_{m}\right]^{2}\right\}^{1 / 2} .
$$

4. Etude des $\langle\boldsymbol{Y}(\boldsymbol{m}, \boldsymbol{j})\rangle_{m}$ et $\sigma_{Y}(j)$. - Les figures $5 a$ et $b$ représentent l'allure des histogrammes de $\langle Y(m, j)\rangle_{m}$ pour les mois d'avril et de septembre. En ordonnée, on a par paliers le pourcentage des journées du mois considéré dont le $\langle Y(m, j)\rangle_{m}$ est compris entre les abscisses des extrémités du palier correspondant. On remarque que le pourcentage des journées de relativement fort ensoleillement ou de relativement faible ensoleillement,

$$
\left(\left|\langle Y(m, j)\rangle_{m}\right| \simeq 0,3\right),
$$

Fig. 4. - Coefficients de corrélation d'ordre $p$ pour les mois d'avril ( $a$ ), de juillet $(b)$ et de décembre (c).

[Correlation coefficients of order $p$ for April (a), July (b) and December $(c)$.] 


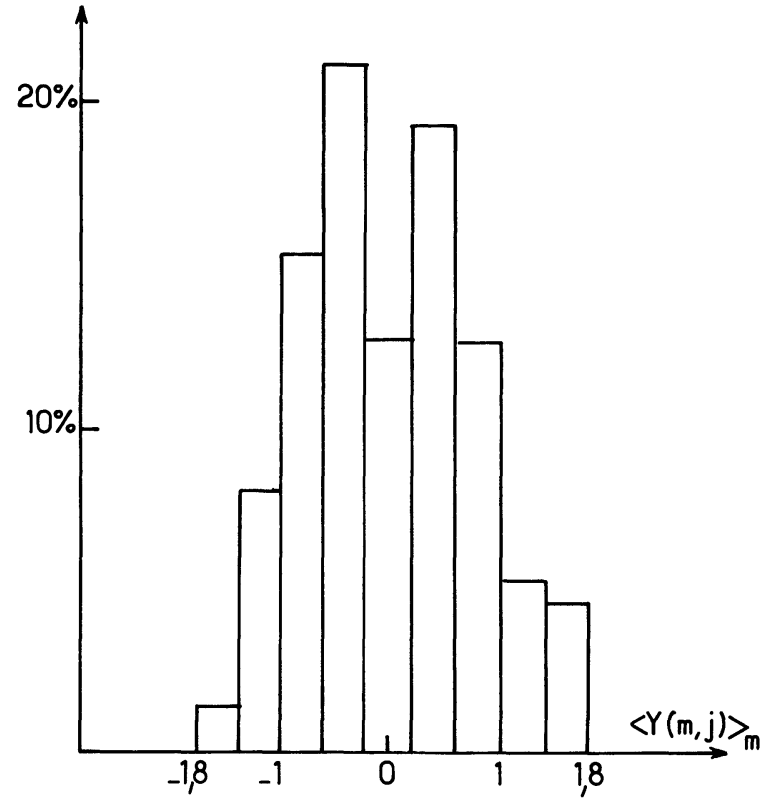

a)

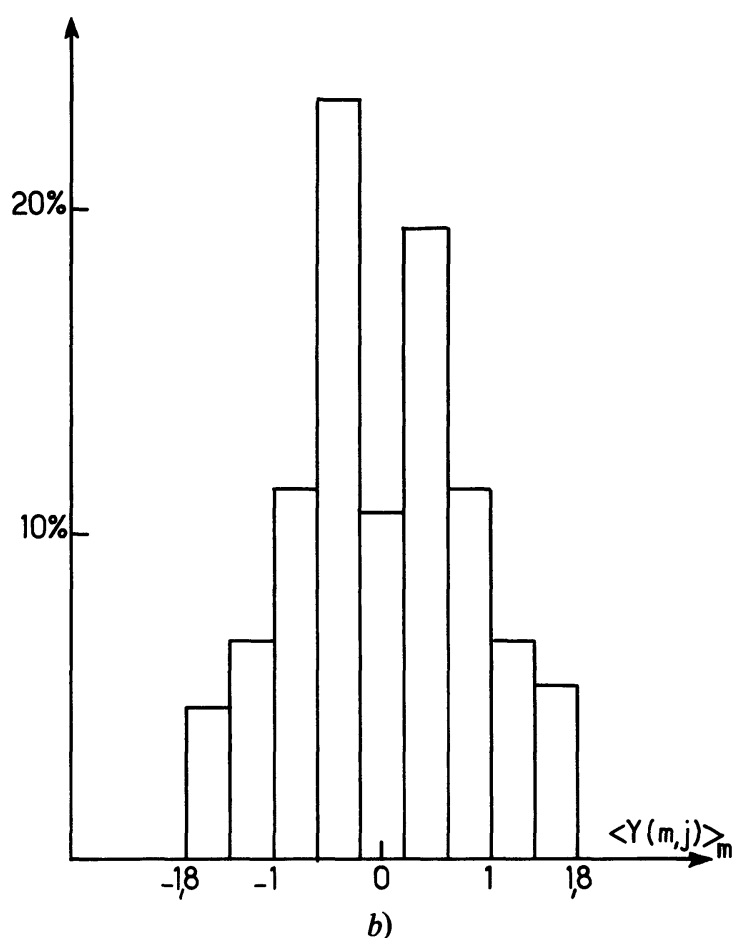

Figs. $5 a, b .-$ Histogrammes relatifs à la variable $Y(m, j)$ pour les mois d'avril (a) et septembre (b).

[Histogramms of $Y(m, j)$ for April (a) and September (b).]

est plus grand que celui des journées à ensoleillement moyen $\left(\langle Y(m, j)\rangle_{m} \simeq 0\right)$. Mais les journées à $\langle Y(m, j)\rangle_{m}$ élevé ne sont pas forcément des journées à apport corrélatif important ; en effet, si les $Y(m, j)$ relatifs à cette journée varient fortement autour de leur moyenne $\left(\sigma_{Y}(j)\right.$ supérieur à $\left.\left|\langle Y(m, j)\rangle_{m}\right|\right)$, l'apport corrélatif correspondant peut être comparable à celui d'une journée de $\langle Y(m, j)\rangle_{m}$ plus faible si $Y(m, j)$ varie très peu autour de cette moyenne.
$\left(\sigma_{Y}(j)\right.$ faible devant $\left.\left|\langle Y(m, j)\rangle_{m}\right|\right)$. On voit donc apparaître l'importance des deux facteurs $\sigma_{Y}(j)$ et $\langle Y(m, j)\rangle_{m}$, ainsi que de leur comparaison. Nous avons représenté, mois par mois, les figures constituées des points $M(j)$ d'abscisse $\langle Y(m, j)\rangle_{m}$ et d'ordonnée $\sigma_{\mathrm{Y}}(j)$ et on a représenté également les deux demidroites $\mathrm{D}_{1}$ et $\mathrm{D}_{2}$ d'équation : $\sigma_{Y}(j)=\left|\langle Y(m, j)\rangle_{m}\right|$. Dans la suite nous dirons qu'une journée $j$ est stable si $\sigma_{Y}(j)<\left|\langle Y(m, j)\rangle_{m}\right|$ (point $M(j)$ correspondant en dessous de $D_{1}$ ou $D_{2}$ ) et instable si

$$
\sigma_{\mathbf{Y}}(j)>\left|\langle Y(m, j)\rangle_{m}\right|
$$

(point $\mathrm{M}(j)$ au-dessus de $\mathrm{D}_{1}$ ou $\mathrm{D}_{2}$ ).

4.1 ETUDE Des NUAGES OBTENUS ET DE LEUR ÉVOLUTION SUIVANT LES MOIS. - On a regroupé les mois qui donnent le même type de figure :

- Mois de janvier, février, novembre et décembre : Pour ces 4 mois d'hiver, on voit apparaître une figure à peu près semblable à celle de la figure $6 a$ (mois de janvier). Les journées de faible ensoleillement $\left.\left(\langle Y(m, j)\rangle_{m}\right\rangle-0,5\right)$ sont très stables. Les journées d'ensoleillement moyen $\left(-0,5<\langle Y(m, j)\rangle_{m}<0,5\right)$ sont très instables et l'instabilité subsiste pour les journées légèrement plus ensoleillées (dissymétrie du nuage).

- Mois de mars, avril, mai, juin et octobre : Pour ces mois intermédiaires, la figure $6 b$ (mois d'avril) montre que les journées de fort ensoleillement $\left.\left(\langle Y(m, j)\rangle_{m}\right\rangle 1\right)$ ont un caractère stable, même remarque pour les journées de faible ensoleillement $\left(\langle Y(m, j)\rangle_{m}<-1\right)$. En revanche, les journées d'ensoleillement moyen $\left(-0,5<\langle Y(m, j)\rangle_{m}<0,5\right)$ présentent une grande instabilité.

- Mois de juillet, août et septembre : Aux mois d'été, correspond une figure symétrique de celle des mois d'hiver (cf. Fig. $6 c$ : mois de juillet).

\subsection{ETUde DU NUAGE OBTENU EN SUPERPUSANT LES} NUAGES CORRESPONDANT A CHAQUE MOIS. - On voit que les journées d'ensoleillement extrême sont en majorité stables et les journées d'ensoleillement moyen instables (cf. Fig. 7). Ceci explique la grande corrélation obtenue à tous les ordres, et il serait intéressant de reprendre le calcul des coefficients de corrélation en séparant les journées d'un mois en 3 groupes :

- journées de fort ensoleillement

- journées de moyen ensoleillement

- journées de faible ensoleillement.

Malheureusement, en effectuant une séparation triple, il resterait dans chaque catégorie trop peu de journées $(\simeq 50)$ et l'erreur commise sur l'évaluation des coefficients de corrélation serait trop importante.

Nous avons donc choisi une séparation en seulement 2 sous-ensembles :

sous-ensemble B :(beau temps)

sous-ensemble $\mathrm{M}$ : (mauvais temps). 


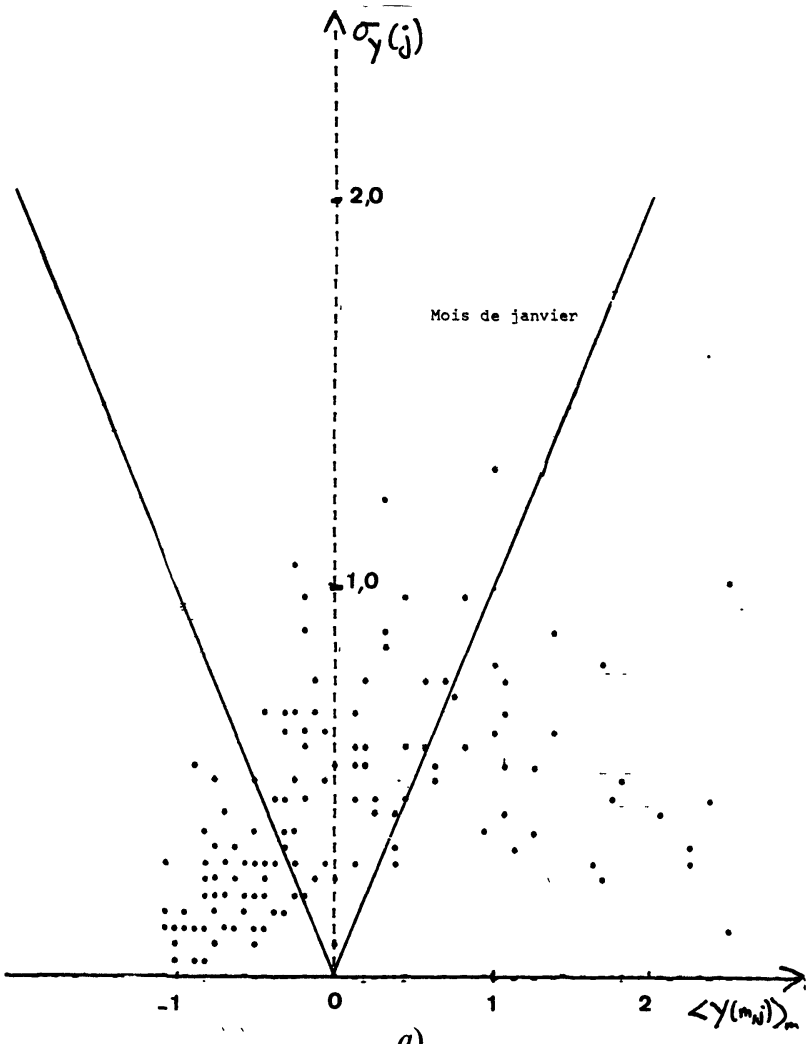

a)

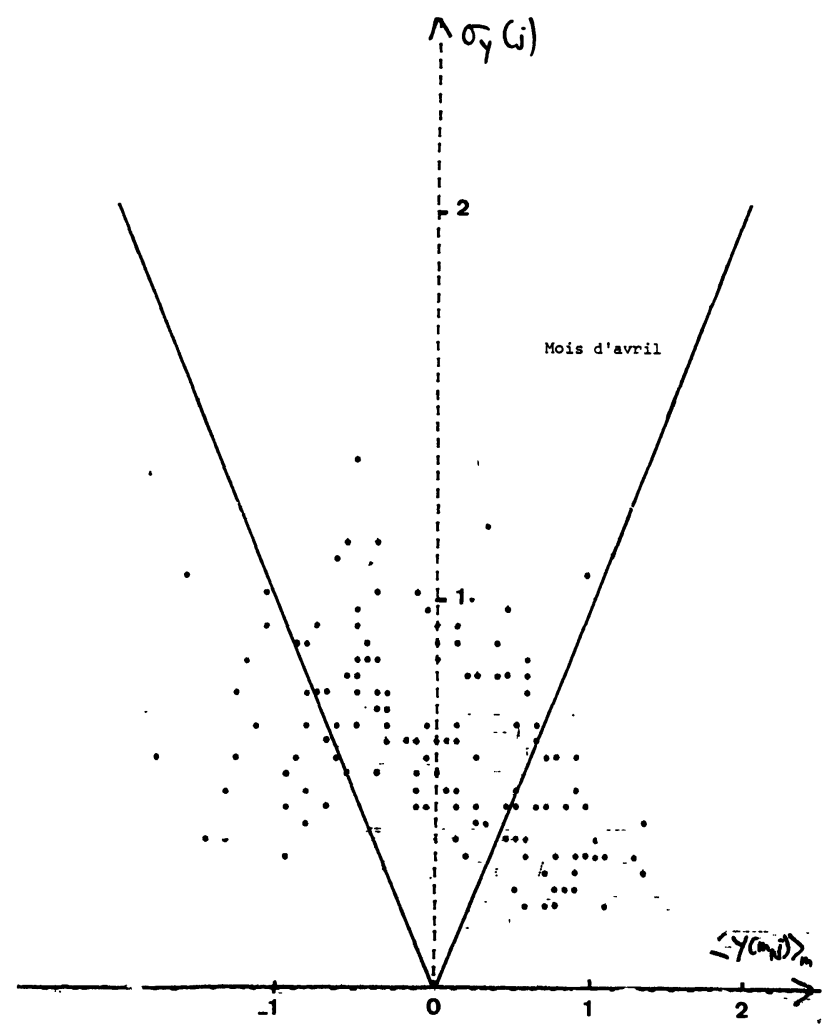

b)

Figs. $6 a, b, c$. - Points $\mathrm{M}(j)$ : d'abscisse $\langle Y(m, j)\rangle_{m}$ et d'ordonnée $\sigma_{Y}(j)$ pour les jours du mois de janvier $(a)$, d'avril $(b)$ et de décembre $(c)$. (150 points par mois.)

[Points $\mathrm{M}(j)$ (coordinates $\langle Y(m, j)\rangle$ and $\left.\sigma_{Y}(j)\right)$ for the days of January $(a)$, April $(b)$ and December $(c)$ (150 points each month).]

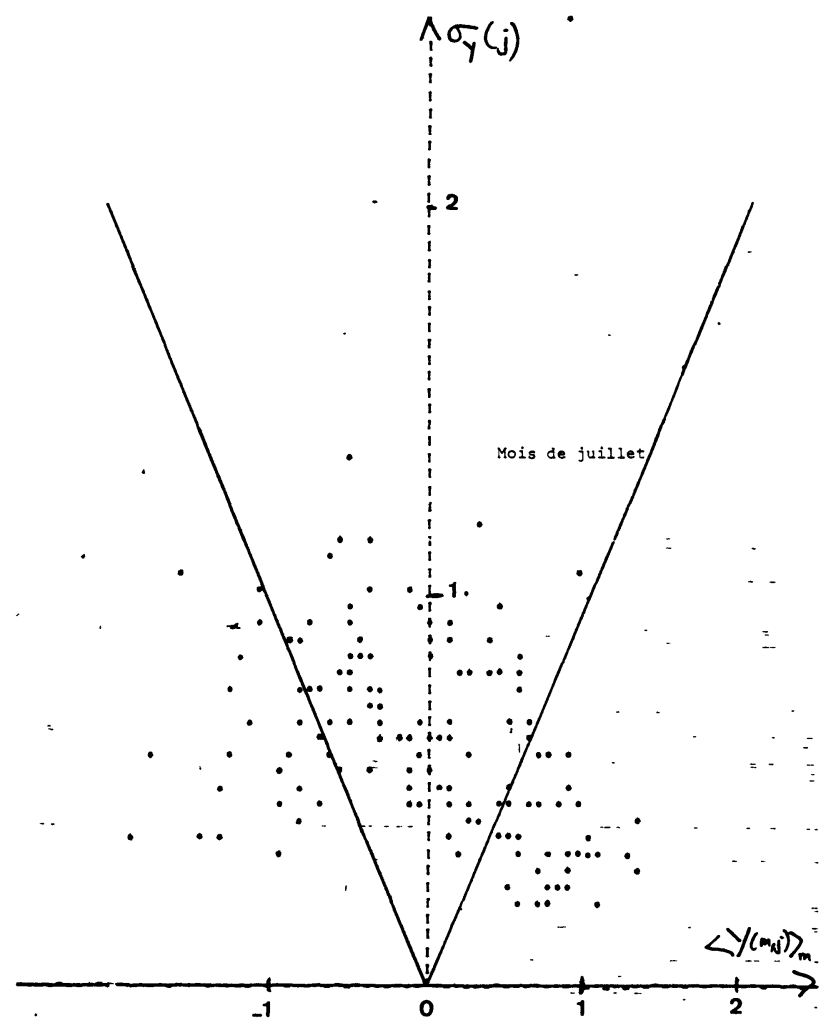

c)

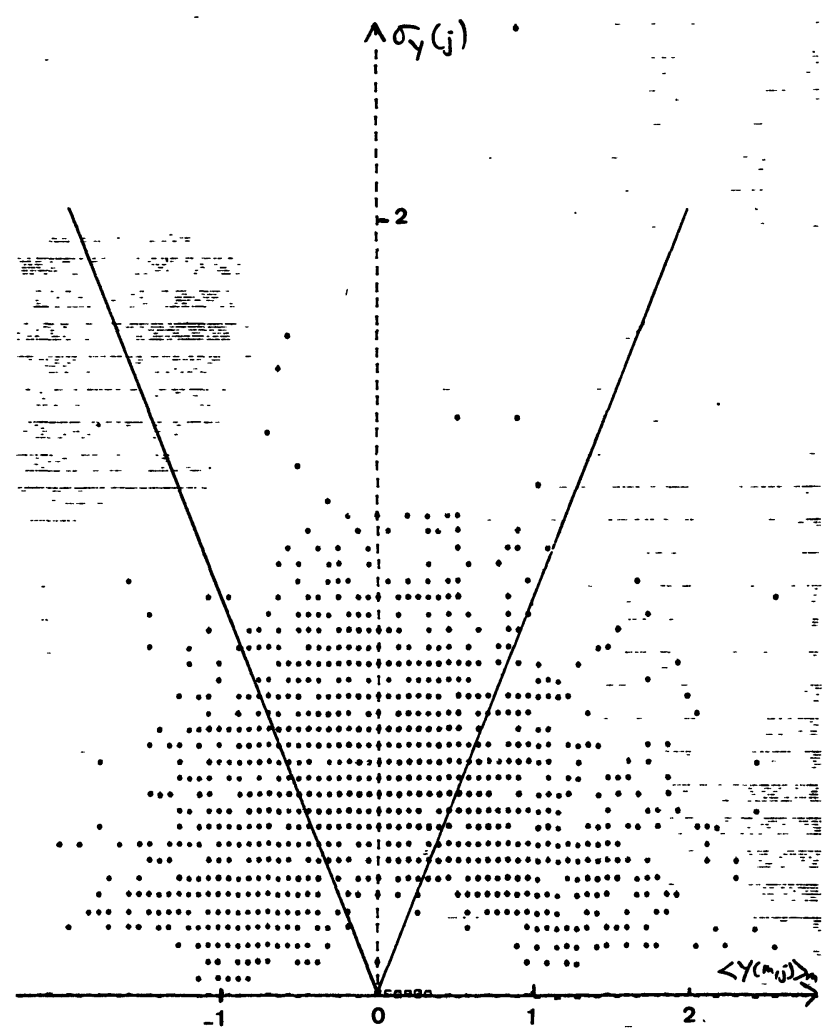

Fig. 7. - Superposition des nuages de points $\mathbf{M}(j)$ relatifs aux 12 mois (1 800 points).

[Union of the sets of points $\mathbf{M}(j)$ for the twelve months (1 800 points).] 
Nous dirons qu'une journée $j$ est de type B si $\left.\langle Y(m, j)\rangle_{m}\right\rangle 0$. De même, une journée $j$ est de type M si $\langle Y(m, j)\rangle_{m}<0$.
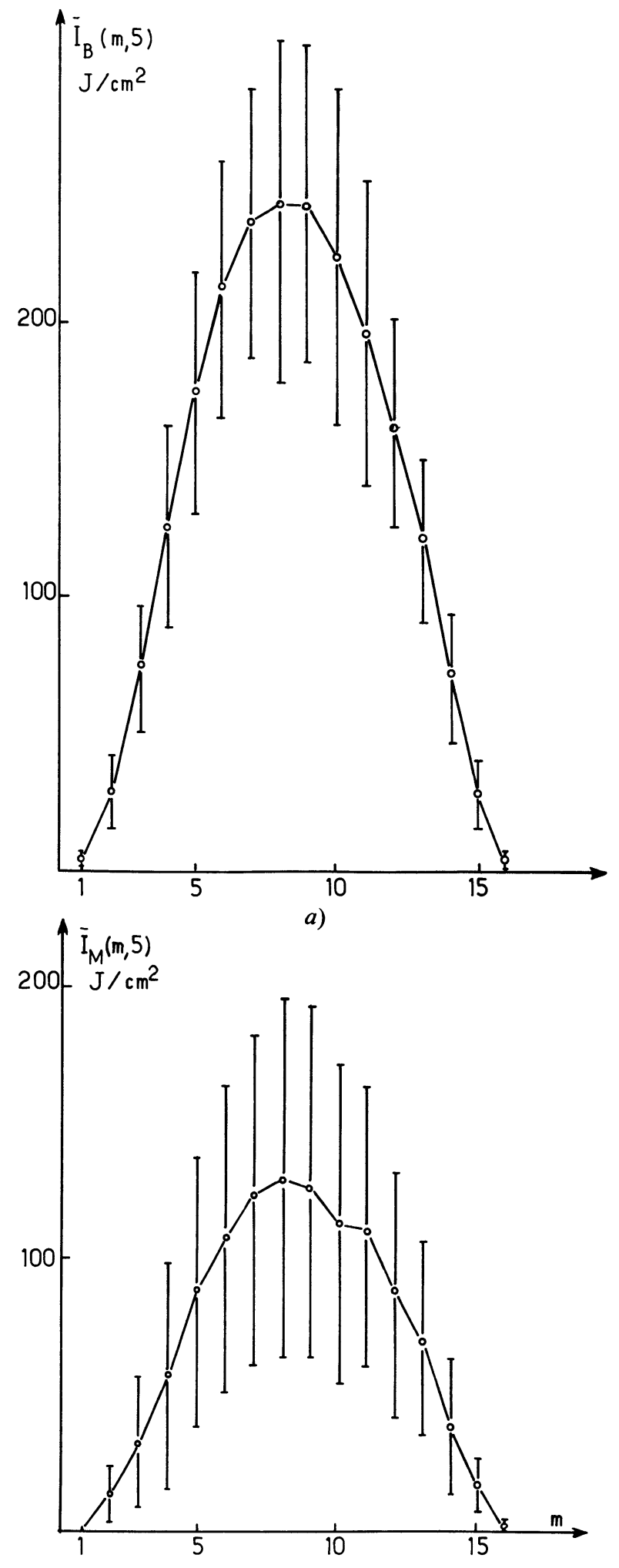

b)

Figs. $8 a, b$. - Valeur moyenne de $I(m, j)$, pour 1 heure donnée $m$, prise sur toutes les journées de type $\mathbf{B}(a)$ ou de type $\mathbf{M}(b)$ du mois de mai avec écart-type.

[Mean value and standart deviation of $I(m, j)$ for a given hour $m$ for the two kinds of weather : B $(a)$ and $\mathbf{M}(b)$.]
5. Séparation en deux types de temps. - Nous allons donc étudier chacun des sous-ensembles B et M séparément, et ceci toujours mois par mois.

Il faut, sur chacun de ces ensembles, redéfinir la variable à étudier, pour les mêmes raisons que celles qui nous avaient conduits à définir $Y(m, j)$ par (3); nous définirons pour une journée du sous-ensemble $B$ du mois $k$ la variable $Y_{\mathbf{B}}(m, j)$ par :

$$
Y_{\mathrm{B}}(m, j)=\frac{I(m, j)-\bar{I}_{\mathrm{B}}(m, k)}{\sigma_{\mathrm{B}}(m, k)}
$$

$\bar{I}_{\mathrm{B}}(m, k)$ étant la moyenne pour l'heure $m$ des $I(m, j)$, moyenne prise sur les $j$ du sous-ensemble B du mois $k$ (journées de beau temps du mois $k$ ), $\sigma_{\mathrm{B}}(m, k)$ étant calculé comme $\sigma(m, k)$ (cf. formule (2)), mais en se limitant également aux journées de type $B$ du mois $k$.

Définitions analogues pour le sous-ensemble $M$ et pour un mois donné $k$ (il suffit de remplacer B par $\mathbf{M}$ dans les définitions précédentes)

$$
Y_{\mathrm{M}}(m, j)=\frac{I(m, j)-\bar{I}_{\mathrm{M}}(m, k)}{\sigma_{\mathrm{M}}(m, k)}
$$

$j$ étant une journée de type $M$ du mois $k$.

Les résultats relatifs au mois de mai $(k=5)$ sont donnés par les figures $8 a$ et $8 b$ qui représentent pour ce mois :

$\bar{I}_{\mathbf{B}}(m, k), \quad \sigma_{\mathbf{B}}(m, k) \quad$ puis $\quad \bar{I}_{\mathbf{M}}(m, k)$ et $\sigma_{\mathrm{M}}(m, k)$.

De même qu'avant séparation, nous définissons à présent pour chaque journée une moyenne et un écarttype journaliers de la variable réduite correspondante qui sont notés $\left\langle Y_{\mathrm{B}}(m, j)\right\rangle_{m}$ et $\sigma_{Y_{\mathrm{B}}}(j)$ ou $\left\langle Y_{\mathrm{M}}(m, j)\right\rangle_{m}$ et $\sigma_{Y_{\mathrm{M}}}(j)$ suivant que $j \subset \mathrm{B}$ ou $j \subset \mathrm{M}$. Nous n'avons pas tracé les histogrammes correspondant à ces valeurs moyennes journalières car après séparation, il reste trop peu de points pour que ces histogrammes soient significatifs. La lecture des nuages de points d'ordonnée $\sigma_{Y_{\mathrm{B}}}(j)$ (resp. $\sigma_{Y_{\mathrm{M}}}(j)$ ) et d'abscisse $\left\langle Y_{\mathrm{B}}(m, j)\right\rangle_{m}\left(\right.$ resp. $\left.\left\langle Y_{\mathrm{M}}(m, j)\right\rangle_{m}\right)$ pour les journées $j$ de type $B$ (resp. $M)$ d'un mois donné est difficile à faire pour les mêmes raisons. Nous avons donc superposé pour chaque type de temps, les nuages correspondant aux différents mois. Dans le cas du sous-ensemble B, pour les journées de faible $\left\langle Y_{\mathbf{B}}(m, j)\right\rangle_{m}$ (valeurs inférieures à $-0,5)$ on observe des $\sigma_{Y_{B}}(j)$ quelconques, au contraire les journées de fort $\left\langle Y_{\mathrm{B}}(m, j)\right\rangle_{m}$ gardent une certaine stabilité (cf. Fig. 9), mais moins marquée qu'avant la séparation de $\mathbf{B}$ et $\mathbf{M}$. On arrive à une conclusion similaire mais symétrique pour les journées de type M (cf. Fig. 10).

5. 1 VÉRIFICATION DE LA STATIONNARITÉ DE $Y_{\mathrm{B}}(m, j)$ ET $Y_{\mathrm{M}}(m, j)$. - Le test de stationnarité est entièrement identique à celui que l'on avait fait avant séparation (voir 2.3). 


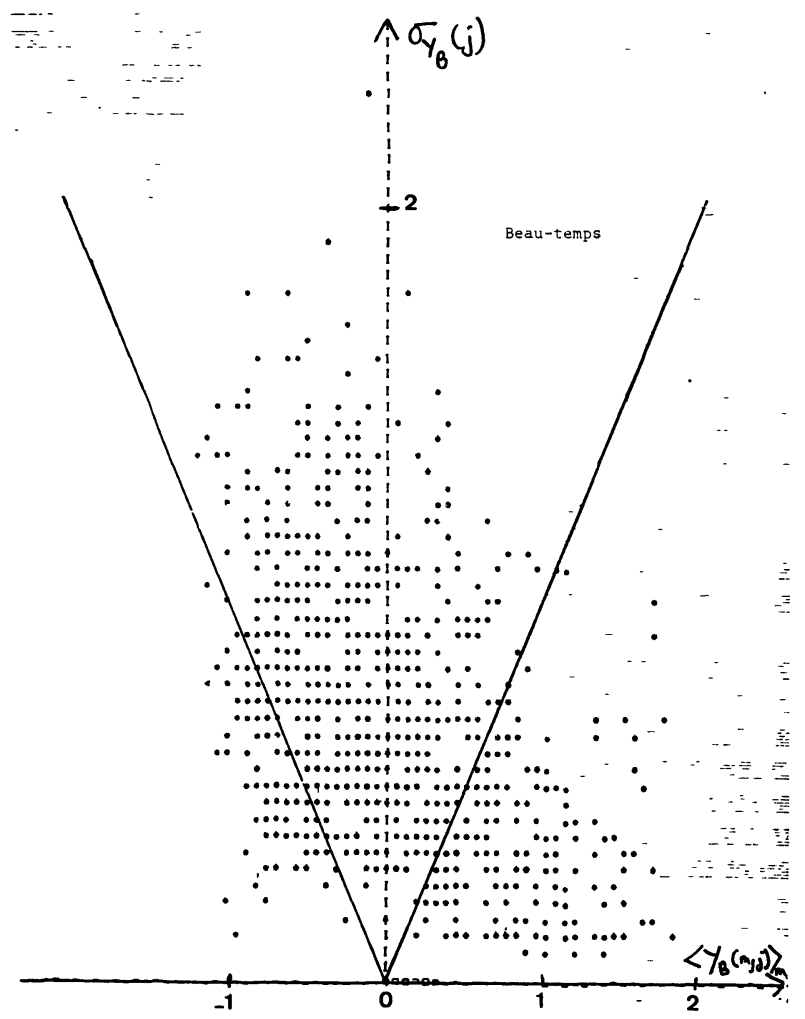

Fig. 9. - Superposition des nuages de points $\mathbf{M}(j)$ relatifs aux jours de temps B pour les 12 mois (890 points).

[Union of the sets of points $\mathrm{M}(j)$ for the days of kind B and the twelve months (890 points).]

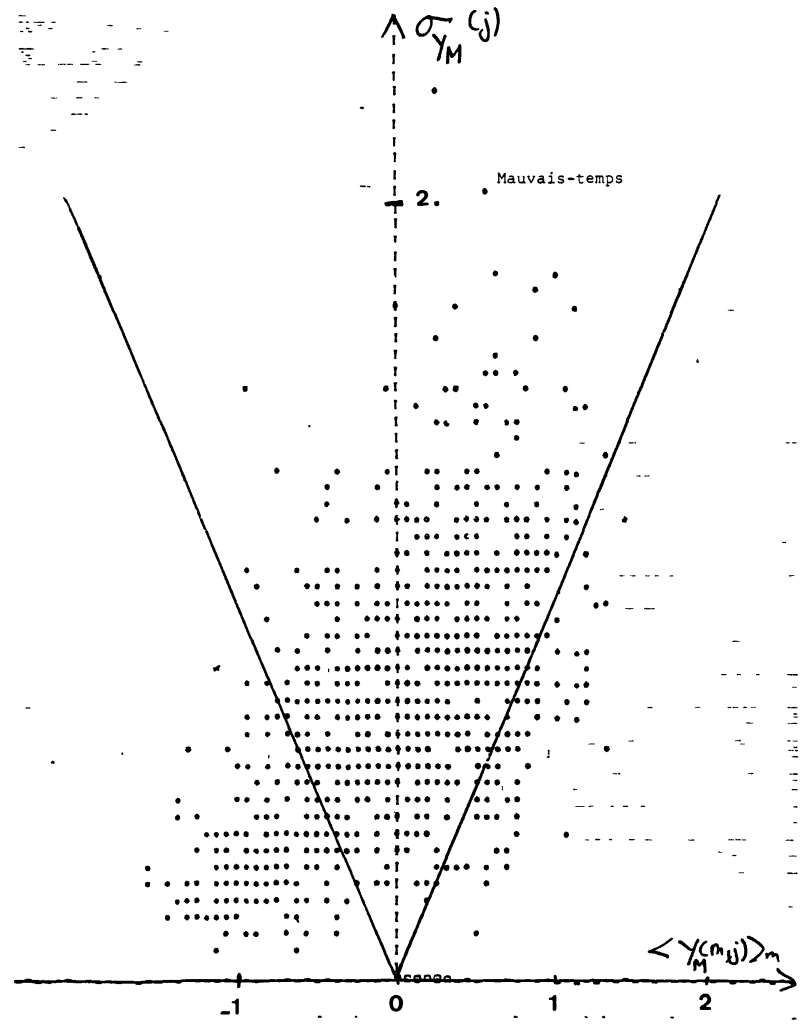

Fig. 10. - Superposition des nuages de points $\mathbf{M}(j)$ relatifs aux jours de temps $M$ pour les 12 mois (910 points).

[Union of the sets of points $\mathbf{M}(j)$ for the days of kind $\mathrm{M}$ and the twelve months (910 points).]
On définit de la même façon le coefficient de corrélation d'ordre $p$ pour l'heure $m$ pour les journées de type $\mathrm{B}$ du mois $k$ par :

$$
\left(r_{p, m}\right)_{\mathbf{B}}=\left\langle Y_{\mathbf{B}}(m, j) Y_{\mathbf{B}}(m+p, j)\right\rangle_{j \subset \mathbf{B}_{k}}
$$

$B_{k}$ représentant l'ensemble des journées de type B du mois $k$.

Définition analogue pour les journées de type $\mathrm{M}$, on remplace B par $\mathbf{M}$.

Dans les deux cas (B ou M), on arrive à une conclusion analogue à celle du (2.3) : l'hypothèse de stationnarité n'est pas contredite par le test.

5.2 TRACÉ DES COURBES DE COEFFICIENTS DE CORRÉLATION MOIS PAR MOIS ET POUR CHAQUE TYPE DE TEMPS. - On définit le coefficient de corrélation d'ordre $p$ pour un type de temps B ou M par :

$$
\left(\rho_{p}\right)_{\mathbf{B}}=\left\langle\left(r_{p, m}\right)_{\mathbf{B}}\right\rangle_{m} \quad \text { pour le sous-ensemble B }
$$
et

$$
\left(\rho_{p}\right)_{\mathbf{M}}=\left\langle\left(r_{p, m}\right)_{\mathbf{M}}\right\rangle_{m} \quad \text { pour le sous-ensemble } \mathbf{M} \text {. }
$$

On obtient dans les 2 cas des courbes ayant la forme d'exponentielles mais décroissant beaucoup plus rapidement qu'avant séparation, ce qui semble bien confirmer les remarques faites dans l'étude de nuages $\sigma_{Y_{\mathbf{B}}}(j)\left(\operatorname{resp} . \sigma_{Y_{\mathbf{M}}}(j)\right)$ en fonction de $\left\langle Y_{\mathbf{B}}(m, j)\right\rangle m$ (resp. $\left.\left\langle Y_{M}(m, j)\right\rangle_{m}\right)$.
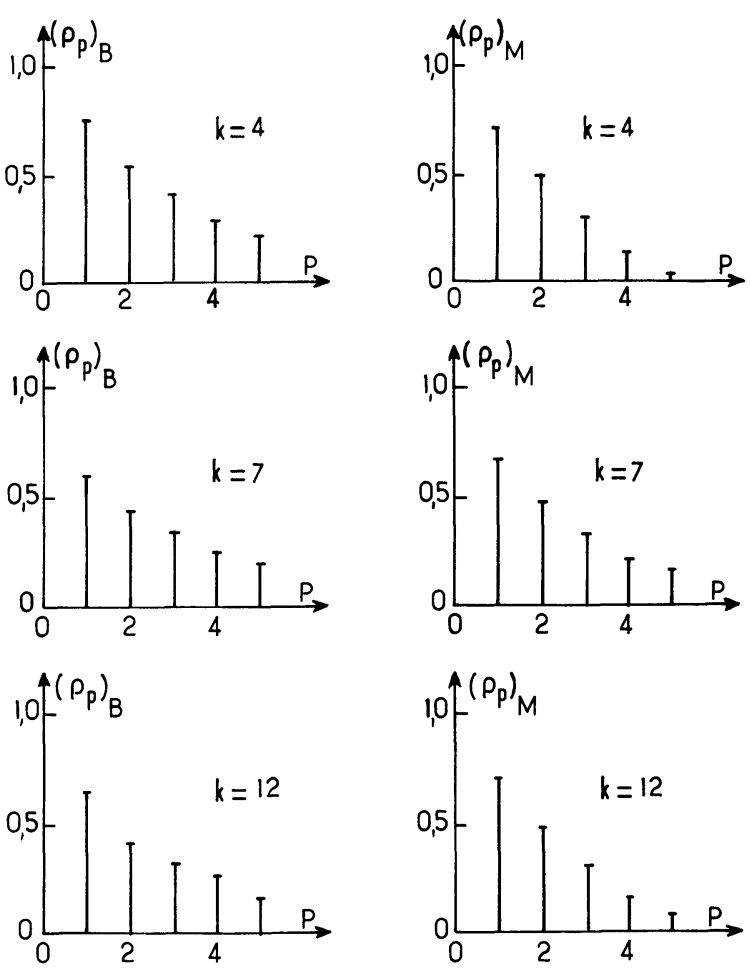

Fig. 11. - Coefficients de corrélation d'ordre $p$ pour les mois d'avril $(k=4)$, de juillet $(k=7)$ et de décembre $(k=12)$ et pour les 2 types de temps.

[Correlation coefficients of order $p$ for both kinds of days, for April $(k=4)$, July $(k=7)$ and December $(k=12)$.] 
La figure 11 représente pour les mois d'avril, de juillet et de décembre et pour chacun des sousensembles $B$ et $\mathbf{M}$ l'allure des coefficients de corrélation d'ordre $p(p=0, \ldots, 5)$.

6. Identification du processus. - Les fonctions d'autocorrélation des divers mois et pour les deux types de temps envisagés ayant l'allure d'exponentielles décroissantes, on peut supposer [8] que le processus est un auto-régressif (A-R). Ce n'est pas un auto-régressif d'ordre 1 car, s'il en était ainsi, le coefficient d'autocorrélation d'ordre $p: \rho_{p}$ serait égal à $\left(\rho_{1}\right)^{p}$, ce qui n'est pas le cas. Les courbes obtenues semblent en fait la combinaison d'au moins deux exponentielles. Nous nous limiterons à l'identification à un processus A-R d'ordre 2 .

C'est-à-dire :

$$
\begin{array}{r}
Y_{\mathbf{B}}(m, j)=\Phi_{1 \mathrm{~B}} Y_{\mathbf{B}}(m-1, j)+\Phi_{2 \mathrm{~B}} Y_{\mathbf{B}}(m-2, j)+ \\
+a(m)
\end{array}
$$

pour une journée de type B, et :

$$
\begin{aligned}
Y_{\mathrm{M}}(m, j)=\Phi_{1 \mathrm{M}} Y_{\mathrm{M}}(m-1, j)+ \\
\quad+\Phi_{2 \mathrm{M}} Y_{\mathrm{M}}(m-2, j)+a(m)
\end{aligned}
$$

pour une journée de type $\mathbf{M}$,

$a(m)$ étant un bruit blanc dont on déterminera plus loin la puissance. Pour alléger l'écriture, on n écrira plus dans la suite $\Phi_{1 \mathrm{~B}}, \Phi_{1 \mathrm{M}}, \Phi_{2 \mathrm{~B}}, \Phi_{2 \mathrm{M}}$ mais simplement $\Phi_{1}, \Phi_{2}$ étant entendu qu'ils sont fonction du type de temps. De même pour les coefficients de corrélation $\left(\rho_{p}\right)_{\mathrm{B}}$ et $\left(\rho_{p}\right)_{\mathrm{M}}$, on écrira simplement $\rho_{p}$. On en tire [8] :

$$
\left(\rho_{p}\right)_{\text {thiorique }}=\frac{G_{1}\left(1-G_{2}^{2}\right) G_{1}^{p}-G_{2}\left(1-G_{1}^{2}\right) G_{2}^{p}}{\left(G_{1}-G_{2}\right)\left(1+G_{1} G_{2}\right)}
$$

où $G_{1}^{-1}$ et $G_{2}^{-1}$ sont les racines de l'équation caractétéristique :

$$
1-\Phi_{1} x-\Phi_{2} x^{2}=0
$$

Pour satisfaire la condition de stationnarité, ces racines doivent se trouver en dehors du cercle unité, ce qui impose aux paramètres $\Phi_{1}$ et $\Phi_{2}$ de se trouver dans la région triangulaire suivante :

$$
\begin{aligned}
\Phi_{1}+\Phi_{2} & <1 \\
\Phi_{2}-\Phi_{1} & <1 \\
-1<\Phi_{2} & <1 .
\end{aligned}
$$

Résultat que l'on devra vérifier.

On a :

$$
\begin{array}{r}
\frac{1}{G_{1} G_{2}}=-\frac{1}{\Phi_{2}} \\
\frac{1}{G_{1}}+\frac{1}{G_{2}}=-\frac{\Phi_{1}}{\Phi_{2}}
\end{array}
$$

ce qui donne les paramètres $\Phi_{1}$ et $\Phi_{2}$ en fonction de $G_{1}$ et $G_{2}$ par :

$$
\left.\begin{array}{l}
\Phi_{1}=G_{1}+G_{2} \\
\Phi_{2}=-G_{1} G_{2}
\end{array}\right\} .
$$

Nous avons déterminé, pour chaque mois et chaque type de temps, $G_{1}$ et $G_{2}$ par une méthode des moindres carrés, utilisant les $\rho_{p}$ que nous avons obtenus à partir des données; nous avons minimisé la somme suivante :

$$
\begin{aligned}
& F\left(G_{1}, G_{2}\right)=\sum_{\rho=1}^{5} \times \\
& \quad \times\left[\frac{G_{1}\left(1-G_{2}^{2}\right) G_{1}^{p}-G_{2}\left(1-G_{1}^{2}\right) G_{2}^{p}}{\left(G_{1}-G_{2}\right)\left(1+G_{1} G_{2}\right)}-\rho_{p}\right]^{2} .
\end{aligned}
$$

Après avoir obtenu $G_{1}$ et $G_{2}$, on en déduit $\Phi_{1}$ et $\Phi_{2}$ par (8).

Les résultats obtenus pour divers mois, pour les deux types de temps (B ou M) sont donnés dans le tableau I.

Tableau I

\begin{tabular}{ccc}
\multicolumn{1}{c}{$\Phi_{1}$} & Beau temps & Mauvais temps \\
$\Phi_{2}$ & $\mathrm{~B}$ & $\mathrm{M}$ \\
- & - & - \\
Avril & 0,754 & 0,875 \\
& $-0,019$ & $-0,191$ \\
Juillet & 0,509 & 0,673 \\
& 0,164 & 0,003 \\
Décembre & 0,612 & 0,844 \\
& 0,056 & $-0,161$
\end{tabular}

Un calcul d'erreur sur les opérations faites permettrait de donner ces résultats à $\Delta \Phi=0,005$ près mais il ne faut pas oublier que les $\rho_{p}$ utilisés ont été obtenus à 0,1 près environ. Nous ne considérons donc pas que ces résultats constitueraient à eux seuls une justification de la séparation B.M. que nous avons faite.

7. Utilisation du modèle pour la simulation. - Dans ce paragraphe, nous décrivons une méthode de simulation de l'ensoleillement au pas de temps de l'heure pour une journée d'un type de temps et d'un mois donnés, à partir des paramètres $\Phi_{1}$ et $\Phi_{2}$ relatifs à ce type de temps et ce mois (indice $\mathrm{B}$ ou $\mathrm{M}$ implicite).

Dans un premier temps, nous obtenons les $Y(m, j)$ (indice $B$ ou $M$ sous-entendu) relatifs à la journée simulée. On résumera les formules (6) et (7) en une seule, à savoir :

$$
Y(m, j)=\Phi_{1} Y(m-1, j)+\Phi_{2} Y(m-2, j)+a(m)
$$

$a(m)$ étant un bruit blanc gaussien dont la puissance se calcule facilement [8] :

$$
\left\langle a^{2}(m)\right\rangle=1-\rho_{1} \Phi_{1}-\rho_{2} \Phi_{2} .
$$


Ce bruit blanc est fourni par un ordinateur. Pour obtenir $Y(m, j)$ à l'aide de la récurrence (9), il faut les 2 valeurs antérieures de $Y: Y(m-1, j)$ et $Y(m-2, j)$. Nous initialisons cette récurrence par 2 valeurs nulles, mais cette initialisation arbitraire donne un transitoire non stationnaire au départ; aussi, nous n'avons pas utilisé les 16 premières valeurs, nous avons admis que l'effet de ce transitoire est amorti après 10 récurrences et nous utilisons les 16 valeurs suivantes.

Ensuite, à partir des 16 valeurs $Y(m, j) m=1, \ldots, 16$ on en déduit 16 valeurs de $I(m, j) m=1, \ldots, 16$ par les formules (4) ou (5) selon le type de temps et le mois considérés.

8. Prévision. - Le but de la prévision, dans le cadre de notre étude est le suivant : se plaçant à une heure donnée, d'un jour et d'un mois donnés, on veut prévoir le mieux possible, l'irradiation globale correspondant à l'heure suivante, tout en connaissant l'irradiation globale correspondant aux heures précédentes.

Mais, comme nous l'avons vu, notre modèle s'applique pour 1 mois donné et surtout pour 1 type de temps donné (B ou M). Pour faire une prévision de l'irradiation, nous devons donc d'abord prévoir le type de temps.

Toutefois, pour tester la validité du modèle, nous allons travailler sur des journées déjà passées et dont on sait si elles sont de type B ou M. Par exemple, soit une journée de type $\mathrm{B}$ du mois $k$, nous sommes à l'heure $m$ et cherchons à prévoir l'heure $(m+1)$. Avec notre modèle, le meilleur estimé en moyenne quadratique est alors :

$$
Y_{\mathbf{B}}(m+1, j)=\Phi_{1 \mathbf{B}} Y_{\mathbf{B}}(m, j)+\Phi_{2 \mathrm{~B}} Y_{\mathbf{B}}(m-1, j)
$$

d'où l'on tire :

$$
\begin{aligned}
I(m+1, j)=Y_{\mathbf{B}}(m+1, j) \sigma_{\mathbf{B}}(m+ & 1, k)+ \\
& +\bar{I}_{\mathbf{B}}(m+1, k) .
\end{aligned}
$$

Ce type de prévision, avec a priori, sera appelé dans la suite prévision de type $\mathrm{X}$. Cette prévision est inutili- sable dans la pratique, car on ne connait pas le type (B ou M) de la journée; nous allons décrire une autre méthode de prévision que nous appellerons prévision de type $Z$.

Comme on ne peut prévoir que des heures correspondant à $m \geqslant 3$ (car il est nécessaire de connaître les $2 Y$ précédents), on dispose d'observations $I(m, j)$ antérieures; pour chacune de ces heures antérieures, on calculera la variable $Y(m, j)=\frac{I(m, j)-\bar{I}(m, k)}{\sigma(m, k)}$ $(\bar{I}(m, k)$ et $\sigma(m, k)$ ayant été calculés une fois pour toutes par (1) et (2)).

Puis on calcule la moyenne sur $m$ de ces $Y(m, j)$ correspondant aux heures antérieures. $\mathrm{Si}$ cette moyenne est positive (resp. négative), on déclarera momentanément la journée de type B (resp. M) et on fait la prévision de type $\mathrm{X}$ avec le coefficients adéquats. A chaque heure nouvelle, on recommence le test qui permet de classer momentanément la journée de type B ou $\mathrm{M}$.

Pour tester d'un point de vue statistique les prévisions de type $\mathrm{X}$ ou $\mathrm{Z}$, on les a utilisées pour toutes les journées d'un type de temps et d'un mois donnés (à partir desquelles on a établi le modèle), puis, pour une heure donnée $m$, on a calculé sur l'ensemble de ces journées l'écart-type de l'erreur de prévision donné par :

$$
\varepsilon_{\mathrm{B}}(m, k)=\left\langle\left[I_{p}(m, j)-I(m, j)\right]_{-}^{2}\right\rangle_{j \subset \mathrm{B}_{k}}
$$

pour les jours de type B de $E_{k}, I_{p}(m, j)$ étant l'irradiation prévue pour l'heure $m$ du jour $j$.

De la même façon nous avons calculé l'écart-type $\varepsilon_{\mathrm{M}}(m, k)$ de l'erreur de prévision à l'heure $m$ pour les jours de type $\mathrm{M}$ des mois $k$.

Le tableau II représente pour avril, juillet et décembre (pour les valeurs accessibles de $m$ ) les rap-

\begin{tabular}{|c|c|c|c|c|c|c|c|c|c|c|c|c|c|c|c|}
\hline & & $\mathbf{m}$ & 3 & 4 & 5 & 6 & 7 & 8 & 9 & 10 & 11 & 12 & 13 & 14 & 15 \\
\hline \multirow{2}{*}{ avril } & B & $\begin{array}{l}x \\
z\end{array}$ & - & $\begin{array}{l}0,61 \\
0,63\end{array}$ & $\begin{array}{l}0,61 \\
0,69\end{array}$ & $\begin{array}{l}0,58 \\
0,65\end{array}$ & $\begin{array}{l}0,60 \\
0,65\end{array}$ & $\begin{array}{l}0,76 \\
0,83\end{array}$ & $\begin{array}{l}0,7.8 \\
0,80\end{array}$ & $\begin{array}{l}0,66 \\
0,71\end{array}$ & $\begin{array}{l}0,78 \\
0,81\end{array}$ & $\begin{array}{l}0,73 \\
0,77\end{array}$ & $\begin{array}{l}0,60 \\
0,62\end{array}$ & $\begin{array}{l}0,65 \\
0,67\end{array}$ & $\begin{array}{l}0,68 \\
0,68\end{array}$ \\
\hline & M & $\begin{array}{l}x \\
z\end{array}$ & - & - & $\begin{array}{l}0,65 \\
0,68\end{array}$ & $\begin{array}{l}0,62 \\
0,65\end{array}$ & $\begin{array}{l}0,73 \\
0,76\end{array}$ & $\begin{array}{l}0,76 \\
0,79\end{array}$ & $\begin{array}{l}0,67 \\
0,73\end{array}$ & $\begin{array}{l}0,73 \\
0,75\end{array}$ & $\begin{array}{l}0,78 \\
0,80\end{array}$ & $\begin{array}{l}0,79 \\
0,81\end{array}$ & $\begin{array}{l}0,72 \\
0,74\end{array}$ & $\begin{array}{l}0,79 \\
0,79\end{array}$ & $\begin{array}{l}0,86 \\
0,86\end{array}$ \\
\hline \multirow{2}{*}{ juillet } & B & $\begin{array}{l}x \\
z\end{array}$ & $\begin{array}{l}0,68 \\
0,74\end{array}$ & $\begin{array}{l}0,92 \\
1,02\end{array}$ & $\begin{array}{l}0,-76 \\
0,83\end{array}$ & $\begin{array}{l}0,81 \\
0,88\end{array}$ & $\begin{array}{l}0,89 \\
0,99\end{array}$ & $\begin{array}{l}0,75 \\
0,80\end{array}$ & $\begin{array}{l}0,63 \\
0,65\end{array}$ & $\begin{array}{l}0,66 \\
0,69\end{array}$ & $\begin{array}{l}0,68 \\
0,67\end{array}$ & $\begin{array}{l}0,86 \\
1,01\end{array}$ & $\begin{array}{l}0,72 \\
0,72\end{array}$ & $\begin{array}{l}0,89 \\
0,89\end{array}$ & $\begin{array}{l}0,72 \\
0,74\end{array}$ \\
\hline & M & $\begin{array}{l}x \\
z\end{array}$ & - & $\begin{array}{l}0,69 \\
0,75\end{array}$ & $\begin{array}{l}0,58 \\
0,65\end{array}$ & $\begin{array}{l}0,64 \\
0,69\end{array}$ & $\begin{array}{l}0,65 \\
0,70\end{array}$ & $\begin{array}{l}0,66 \\
0,72\end{array}$ & $\begin{array}{l}0,69 \\
0,71\end{array}$ & $\begin{array}{l}0,78 \\
0,82\end{array}$ & $\begin{array}{l}0,84 \\
0,92\end{array}$ & $\begin{array}{l}0,79 \\
0,85\end{array}$ & $\begin{array}{l}0,73 \\
0,73\end{array}$ & $\begin{array}{l}0,80 \\
0,80\end{array}$ & $\begin{array}{l}0,77 \\
0,80\end{array}$ \\
\hline \multirow{2}{*}{ décembre } & B & $\begin{array}{l}\mathrm{x} \\
\mathrm{z}\end{array}$ & - & - & - & - & $\begin{array}{l}0,76 \\
0,76\end{array}$ & $\begin{array}{l}0,80 \\
0,90\end{array}$ & $\begin{array}{l}0,89 \\
0,97\end{array}$ & $\begin{array}{l}0,77 \\
0,80\end{array}$ & $\begin{array}{l}0,71 \\
0,73\end{array}$ & $\begin{array}{l}0,63 \\
0,63\end{array}$ & - & - & - \\
\hline & M & $\begin{array}{l}x \\
z\end{array}$ & - & - & - & - & $\begin{array}{l}0,72 \\
0,98\end{array}$ & $\begin{array}{l}0,64 \\
0,80\end{array}$ & $\begin{array}{l}0,71 \\
0,80\end{array}$ & $\begin{array}{l}0,69 \\
0,66\end{array}$ & $\begin{array}{l}0,73 \\
0,70\end{array}$ & $\begin{array}{l}0,76 \\
0,76\end{array}$ & - & - & - \\
\hline
\end{tabular}
ports :

$$
\begin{array}{ll}
\varepsilon_{\mathrm{B}}(m, k) / \sigma_{\mathrm{B}}(m, k) & (\text { type B) } \\
\varepsilon_{\mathrm{M}}(m, k) / \sigma_{\mathrm{M}}(m, k) & (\text { type } \mathrm{M})
\end{array}
$$

Tableau II. 
Plus ce rapport est faible devant 1 , meilleure est la prévision. On remarque que la prévision de type $\mathrm{X}$ est à peine meilleure que la prévision de type $Z$. Ce résultat peut s'expliquer par une grande stabilité au cours d'une journée du type de temps provisoire tel que nous l'avons défini, ou bien par le fait que les paramètres obtenus pour les 2 types de temps ne sont pas très différents.

On n'observe pas dans le tableau de nette différence entre les 2 types de temps, mais il ne faut pas oublier que les fluctuations relatives sont nettement plus faibles dans le cas du type B (cf. Figs. $8 a$ et $b$ ).

Les rapports obtenus sont assez encourageants si on les compare à ceux obtenus avec le pas de temps de la journée $[4,5]$ et il faut d'ailleurs tenir compte du fait que la séparation en deux types de temps a diminué la variance.

Notons enfin que l'incertitude relative de la prévi- sion $\varepsilon / I_{P}$ est nettement plus faible que les rapports donnés dans le tableau (puisque $\sigma_{\mathrm{B}}$ et $\sigma_{\mathrm{M}}$ sont plus petits que $I_{P}$, surtout vers le milieu de la journée).

9. Conclusion. - En utilisant la variable réduite associée mois par mois à l'ensoleillement horaire global nous avons montré qu'une séparation très simple en 2 types de temps conduit à une modélisation satisfaisante. Avec un plus grand nombre de données on aurait pu envisager une séparation plus fine, mais les coefficients obtenus pour nos deux types de temps n'étant pas très différents (alors que les valeurs moyennes de $I$ correspondantes sont nettement différentes) on peut penser que des séparations plus fines n'apporteraient pas de fortes différences pour les modèles.

\section{Bibliographie}

[1] Exell, R. H. B., The fluctuation of solar radiation in Thailand. Sol. Energy 19 (1976) 549, 554.

[2] BRINKwORTH, B. J., Autocorrelation and stochastic modelling of insolation sequences. Sol. Energy 19 (1977) 343, 347.

[3] GoH, T. N., Statistical study of solar radiation information in an equatorial region (Singapore). Sol. Energy 22 (1979) 105, 111.

[4] Boileau, E. et Guerrier, B., Comparaison de modèles statistiques saisonniers et non saisonniers en météorologie solaire. Météorologie (1979) 115-130.

[5] Guerrier, B., Bolleau, E. et Benard, C., Analyse statistique temporelle de l'irradiation solaire globale quotidienne : modélisation d'une variable réduite à l'aide de modèles stochastiques A.R.M.A. Revue Phys. Appl. 15 (1980) 93102.

[6] Mejon, M. J., Etude des liaisons entre l'irradiation journalière et les variables météorologiques courantes. Contribution à l'analyse et à la simulation de données d'éclairement direct à pas de temps fin. Thèse (1979), Grenoble.

[7] Mustacchi C., V. Cena, M. Rocchi, Stochastic simulation of hourly global radiation sequences. Sol. Energy 23 (1979) $47-51$

[8] Box, G. E. P. et Jenkins, G. M., Time series Analysis, Holden Day (1970). 